REBOA at Role 2 Afloat: Resuscitative endovascular balloon occlusion of the aorta as a bridge to damage control surgery in the military maritime setting

\title{
Rees, Paul
}

(Corresponding Author)

cardiacexpert@,nhs.net

Academic Department of Military Medicine

London, UK

University of St Andrews School of Medicine

St Andrews, UK

\section{Waller, Bentley}

University Hospital Southampton NHS Foundation Trust, Shackleton Department of Anaesthetics Southampton, UK

\section{Buckley, Andrew Martin}

Academic Department of Military Medicine

London, UK

\section{Doran, Catherine}

Royal Centre for Defence Medicine, Department of Surgery

Birmingham, UK

\section{Bland, Steve}

Queen Alexandra Hospital, Department of Emergency Medicine

Portsmouth, Portsmouth, UK

\section{Scott, Tim}

University Hospitals of North Midlands NHS Trust, Department of Anaesthesia and Critical Care Stoke-on-Trent, Staffordshire, UK

\section{Matthews, Jon}

Royal Cornwall Hospitals NHS Trust, Department of Orthopaedics and Trauma Surgery Truro, Cornwall, UK

National Command Headquarters, Clinical Director Role 2 Afloat

Portsmouth, UK 


\begin{abstract}
Role 2 Afloat provides a damage control resuscitation and surgery facility in support of maritime, littoral and aviation operations. REBOA offers a rapid, effective solution to exsanguinating haemorrhage from pelvic and non-compressible torso haemorrhage. It should be considered when the patient presents in a peri-arrest state, if surgery is likely to be delayed, or where the single operating table is occupied by another case. This paper will outline the data in support of endovascular haemorrhage control, describe the technique, and explore how REBOA could be delivered using equipment currently available in the Royal Navy Role 2 Afloat equipment module. Also discussed are potential future directions in endovascular resuscitation.
\end{abstract}

\title{
Key Messages:
}

- REBOA is part of an evolving advanced endovascular resuscitation system.

- REBOA has the potential to offer rapid and effective anatomical control of bleeding, allowing for physiological optimisation before and during damage control surgery.

- In the military setting, and particularly where access to surgery is limited, REBOA could save lives from haemorrhage. 


\section{REBOA at Role 2 Afloat: Resuscitative endovascular balloon occlusion of the aorta as a bridge to damage control surgery in the military maritime setting.}

\section{Introduction}

The Royal Navy Role 2 Afloat (R2A) capability exists to support maritime, littoral and aviation operations by providing damage control resuscitation, including surgery, within 2 hours from the point of wounding according to NATO timelines. The service may be deployed in support of conventional or special forces operations, or as part of a humanitarian and disaster relief response (Figure 1). The system can be rapidly and flexibly delivered from a variety of maritime platforms, some of which have dedicated space pre-allocated, with equipment pre-embarked ready for immediate use.

The majority of deployments utilise a 2-1-2 configuration, comprising of 2 emergency department resuscitation bays, 1 operating theatre table, and 2 level 3 critical care beds. Manning comprises one consultant each from emergency medicine, general surgery and orthopaedics, plus 2 consultants in anaesthesia. The team may be augmented by a consultant physician where a particular requirement is anticipated. The R2A is also equipped with extensive blood products, and basic laboratory facilities to support initial assessment, resuscitation and ongoing ICU care.

\section{Bleeding is the primary cause of death on kinetic combat operations}

The primary aim is to provide a high-readiness emergency surgical facility offering rapid haemorrhage control as part of a strategy of early, effective damage control resuscitation (1) (2). A recent analysis of 4,500 military trauma deaths confirmed that the primary mode of death in combat is haemorrhage, accounting for over $90 \%$ of all deaths. The majority of deaths occur before even reaching a surgical facility (3). A system combining advanced physician-led prehospital care, early blood product delivery and aggressive consultant-delivered acute hospital care has been shown to optimise survival from severe battlefield trauma, despite very complex injury patterns (4). The ongoing development of systems with which to combat bleeding is a top priority in saving life.

Non-compressible torso and pelvic haemorrhage is especially problematic and carries a high case fatality rate (5). Conventional immediate interventions such as pressure dressings, combat tourniquets and haemostatic gauze are unlikely to gain control of bleeding from non-compressible sites. A patient presenting in extremis from this form of bleeding requires effective, immediate proximal haemorrhage control. Whilst it is accepted that the injury patterns seen on recent military operations may differ from those found in future conflicts, bleeding is likely to remain the primary mode of death, as it is within civilian trauma cohorts (6). Swift laparotomy or thoracotomy for aortic cross-clamping, alongside blood product resuscitation will be required. If these are not available, or if there is a delay to gaining surgical control, death will ensue.

In limited-capacity systems, such as the deployed R2A, an alternative endovascular solution which allows anatomical and physiological stabilisation would be of particular value, especially in situations where multiple casualties occur simultaneously, or where a single operating theatre is occupied by a prior case (7).

\section{Resuscitative endovascular balloon occlusion of the aorta}

Resuscitative endovascular balloon occlusion of the aorta (REBOA) is a novel form of haemorrhage control which has been proven to reduce bleeding and improve survival in animal models (8-11) Introduced by a femoral artery sheath, an endovascular balloon is inflated in the aorta, occluding the vessel and acting as an endovascular clamp (Figure 2). REBOA has been demonstrated to be 
efficacious in a large human case series (12). A prospective registry of 114 patients with critical haemodynamic instability in the United States (US) armed forces confirmed that in-hospital REBOA might be a viable alternative to immediate resuscitative thoracotomy (RT) for achieving rapid proximal control. The cohort had an overall mean Injury Severity Score (ISS) of 31.5, and around half suffered traumatic cardiac arrest during initial resuscitation. The study failed to demonstrate any significant difference between REBOA and RT in terms of mortality ( $72 \%$ vs $84 \%$ respectively), but concludes that REBOA is a viable option in centres where the expertise is available (13). A Japanese retrospective observational study comparing REBOA with RT further suggested that in-hospital REBOA is equally effective in terms of adjusted mortality (14). In this series of 259 cases, REBOA was the proximal control strategy in $74 \%$, reflecting important international variations in haemorrhage control strategy. A prospective randomised controlled trial of REBOA is about to commence in the UK, aiming to recruit 120 cases across 4 major trauma centres, in order to provide an analysis of efficacy and cost-effectiveness of the technique. For use within a R2A environment, REBOA is positioned as an additional therapy, to be used where profound instability exists, or where immediate surgical intervention is not possible due to logistical constraints.

Three distinct anatomical aortic landing zones are described; Zone 1 - between left subclavian and coeliac artery, Zone 2 - between coeliac artery and the most caudal renal artery, Zone 3 - between the most caudal renal artery and the aortic bifurcation. In clinical practice, Zones 1 and 3 are used as landing zones for REBOA. Balloon positioning is ideally performed under direct fluoroscopic guidance. However this is not available in a deployed R2A. Indeed, fluoroscopy is frequently not available within conventional emergency departments. Instead, anatomically-derived measurements can be used to insert the balloon catheter to an appropriate depth $(15,16)$, and an anteroposterior X-ray will confirm correct placement.

Once the balloon is in-situ, resuscitation including administration of blood products, and emergency anaesthesia can then be performed. The usual response to REBOA is a brisk, significant rise in systolic blood pressure. REBOA is a temporary anatomical and physiological stabilisation tool, for critically unstable patients with haemorrhagic shock. Its use is suggested where an operating theatre is not immediately available, or where cardiac arrest is imminent. Although currently deployed high-volume infusion devices can often keep pace with severe haemorrhage in the preoperative and anaesthesia phases, there is no evidence that this is a superior strategy to gaining rapid endovascular control. Rapid haemorrhage control might reduce preoperative blood requirements, saving blood products for administration in-theatre or postoperatively once damage control surgery is complete. As well as reducing the multiple adverse effects associated with massive transfusion, in a logistically challenged operation setting this might also conserve valuable blood stocks. Currently available equipment for performing REBOA is listed in Table 1 and shown in Figure 3.

14g Arterial Catheter (Leadercath, Vygon ${ }^{\mathrm{TM}}$ ) - for initial access as a mini-puncture device, prior to exchange for the large-bore sheath. Contains a $16 \mathrm{~g}$, $7 \mathrm{~cm}$ introducer needle, $460 \mathrm{~mm} \mathrm{~J}$-tipped guidewire and $6 \mathrm{~F}, 18 \mathrm{~cm}$ arterial catheter.

Two-Lumen Central Venous Access Set (MAC, Arrow International ${ }^{\mathrm{TM}}$ ) - a two-lumen central access "trauma line" device with a 0.035 " $45 \mathrm{~cm}$ guidewire, dilator and a $10 \mathrm{~cm}$ sheath with a $9 \mathrm{~F}$ distal lumen, suitable for passage of the REBOA balloon.

Embolectomy catheter (Syntel, Applied Medical ${ }^{\mathrm{TM}}$ ) - an endovascular balloon, originally designed for thrombus retrieval. The $7 \mathrm{~F}$ catheter is $80 \mathrm{~cm}$ long, with the balloon capable of expanding to $15 \mathrm{~mm}$ once inflated with $2.5 \mathrm{ml}$ of fluid. 
Table 1: The following REBOA items exist within the current equipment module for R2A.

\section{Suggested process}

The technique can easily be adapted for different clinical environments, or differing skillsets, and can be adjusted to suit the available equipment. This outline specifically refers to equipment carried within the maritime R2A, but could apply to any deployed military unit holding the same equipment (Box 1).

Preparation and arterial cannulation

The REBOA operator should brief the team and prepare the equipment. The primary stage is identification of an arterial access site. The right common femoral artery is the most common, and easiest for the right handed operator. The femoral pulse should be sought, using conventional anatomical landmarks. Once identified ultrasound should be used to confirm the anatomy. Following skin preparation and draping, local anaesthesia infiltration can be performed if required, aiming for vessel entry $2 \mathrm{~cm}$ below the inguinal ligament. A generous stab incision should be made at the insertion site, to facilitate passage of the devices through the skin, avoiding damage to their leading edges. Using the $18 \mathrm{~g}$ introducer needle from the MAC ${ }^{\mathrm{TM}}$ line set, advancing at $45^{\circ}$ to the skin, the femoral artery should be punctured, using an open-needle technique (i.e. no needle attached). Brisk pulsatile flow of arterial blood should usually be observed. Fix the needle firmly in place. Insert the J-tipped $45 \mathrm{~cm} 0.035$ " MAC ${ }^{\mathrm{TM}}$ guidewire to at least half its length. The wire should pass easily. Resistance, or a gritty sensation indicates that the wire is in a dissection flap, or possibly kinked carefully remove the wire, confirm arterial flow from the needle, reposition if necessary. Once the wire is in-situ, remove the needle over the wire, leaving the wire in the vessel. At this point, take the Leadercath ${ }^{\mathrm{TM}}$ sheath and insert this fully into the artery, over the wire. Ultrasound at this point may be used to confirm that the sheath and wire are still sited in the vessel. Remove the wire and confirm that free- aspiration of blood is possible. This step has been included to increase operator confidence that the lumen has been achieved, prior to committing to dilation for the larger MAC ${ }^{\mathrm{TM}}$ line - an experienced endovascular operator may choose to omit this. Next, remove the Leadercath taking care to leave the guidewire in place. Advance the MAC line over the wire, ideally with an assistant fixing the back end wire to avoid kinking. Insert the MAC line fully, remove the dilator and guidewire together. Aspirate, flush and clamp the side ports. In the case of difficulty gaining access, a limited cut-down though a vertical incision over the access site can assist. Blunt digital dissection is all that is required to identify the femoral artery, which can then be cannulated under direct vision. The contralateral groin offers an alternative route.

Insertion of balloon

Remove the Syntel balloon catheter from its housing. Attach a $5 \mathrm{ml}$ syringe filled with $0.9 \%$ saline or a 50:50 mix of saline and iodinated contrast (to enhance visibility on subsequent X-ray). Aspirate air from the balloon. Do not test the balloon as this will make it bulky and prone to damage. Use external landmarks to estimate catheter length to the desired landing zone. For Zone 1 estimate catheter length from the femoral insertion site to midway between suprasternal notch and xiphoid process. A Zone 3 deployment is estimated from the femoral access site to the umbilicus $(15,16)$. In a critically unstable patient with torso trauma, initial deployment in Zone 1 is advised. Zone 3 should be considered as an initial REBOA strategy where pelvic trauma is believed to be the primary source of bleeding. Pass the balloon carefully through the MAC line valve, using the $10 \mathrm{~cm}$ incremental markers to guide depth of insertion. The balloon should pass freely, without resistance. If 
resistance if felt, withdraw the balloon to the sheath and gently attempt again. Once at the desired depth, open the 3-way tap and deliver $2.5 \mathrm{ml}$ of fluid from the syringe, stopping if resistance is felt (Figures 4 and 5). Turn the 3-way tap to isolate the balloon from the delivery syringe. Manually hold the balloon in place, as any cardiac output will tend to displace the balloon caudally. The sheath should be sutured firmly in place.

\section{Subsequent management}

A proximal systolic pressure increase of $\sim 50 \mathrm{mmHg}$ is usual. Volume expansion can be optimised if required, whilst preparations for surgery are made. Time is now key - REBOA offers a temporary solution and a bridge to damage control surgery. Prolonged REBOA has been shown to increase circulating levels of lactate, interleukin-6, and an increase in vasopressor requirements (9). Partial REBOA (pREBOA), allowing a limited amount of forward flow, is more physiologically and haemodynamically tolerable option and might further extend the "golden hour" after traumatic injury $(17,18)$. Pragmatically, an initial complete REBOA inflation, to achieve fast control is advocated. If stability is achieved, and the up-stream pressures are supranormal, controlled partial deflation and PREBOA is reasonable, and associated with better outcomes (19). Although this appears complex in a text description, it is highly intuitive and easily learned in a workshop setting. A radial arterial pressure line would be of value; if the pressure is supranormal, cautious balloon deflation by removing $0.5 \mathrm{ml}$ aliquots of fluid could be considered - obviously at the expense of some downstream bleeding. An X-ray should be acquired if transfer to theatre is not immediate, to confirm balloon positon. The level of $L 2$ usually indicates the level of the renal arteries - a Zone 1 balloon should thus be positioned above this. The level of $L 4$ usually represents the aortic bifurcation, hence a Zone 3 inflation should be positioned between $L 4$ and $L 2$.

\section{Removal}

The REBOA sheath and balloon should be monitored closely once deployed, taking care to avoid accidental dislodgement. A team member should be tasked with maintaining balloon position, as caudal migration will become likely as cardiac output increases following resuscitative measures. Once in the operating theatre, close liaison between anaesthetist and surgeon should take place regarding the timing of balloon deflation and removal. This would usually be considered during or after initial damage control surgery, and a cautious, step-wise balloon deflation, (e.g. $0.5 \mathrm{ml}$ at a time) is proposed. If haemodynamics remain favourable, and haemostasis is achieved, balloon removal can occur. Prolonged presence of an undeployed balloon catheter and/or arterial sheath should be avoided - thrombus can easily form and compromise limb viability. Fully deflate the balloon. Gently remove the balloon catheter through the MAC line. Three options now exist; remove the line and perform direct manual pressure over the access site for 10 minutes, remove the line with proximal vascular control and perform an arteriorrhaphy, or use a fascial suture to close the vessel. As the device is $9 \mathrm{~F}$, the direct pressure option is possible, though in the setting of a potentially coagulopathic polytrauma patient there is a risk of further bleeding. Also, the ability to test for coagulopathy might be suboptimal in the deployed maritime setting. Formal arterial repair be time consuming, and might be outwith the usual practice of some surgeons. The fascial suture technique, closing the cribriform fascia with a horizontal mattress suture applying compression over the point of insertion whilst the vessel is controlled with sloops is easy, fast and associated with a very low long term complication rate-(20) (20).

\section{Other potential settings and developments}


REBOA could easily be applied in other military settings where access to an operating table might be compromised. This could include use by the Commando Forward Surgical Group in support of littoral operations by the Royal Marines, or by British Army Role 2 Light Manoeuvre units.

Prehospital REBOA has been reported (21), delivered by physicians of Londons Air Ambulance. The procedure could easily be adopted for use by military Medical Emergency Response Teams physicians, and early prehospital use is likely to deliver the most overall clinical benefit. A device, intended specifically for fluoroscopy-free deployment, is now approved for use in Europe (ER-REBOA catheter, Prytime Medical ${ }^{\mathrm{TM}}$ ), which might provide a more deliverable option for deployed use.

REBOA offers a bridge to damage control surgery (22). If initial stabilisation fails or cardiac arrest ensues, presence of an aortic catheter extends the options to include therapies such as selective aortic arch perfusion (SAAP) with blood or novel resuscitation fluids (23) (23). If initial efforts still fail, rapid cooling with ice-cold saline boluses, and conversion to full cardiopulmonary bypass is possible. This combination of techniques - emergency preservation and resuscitation (EPR) for cardiac arrest from trauma -- is currently under investigation (24). It is likely that REBOA, SAAP and EPR will merge to form a hybrid advanced endovascular toolkit for severe haemorrhagic shock (25). A sound military REBOA programme is the first step towards exploiting these techniques to save lives in future.

\section{Formal training courses}

The Endovascular Hybrid Trauma and Bleeding Management (EVTM) workshop runs twice annually in Orebro, Sweden. The course offers theoretical training, simulator sessions and live tissue work using an animal model of severe trauma. It allows candidates to gain real-time, hands-on experience of performing REBOA, as well as a variety of other endovascular techniques such as stent-grafting, coiling and use of haemostatic glue, under the supervision of an expert multispecialty international faculty. The Basic Endovascular Skills for Trauma (BEST) course, operated by the American College of Surgeons Committee offers an alternative, designed as a means to offering basic endovascular techniques to surgeons who have limited or no formal endovascular training.

\section{Conclusions}

REBOA offers a rapid, effective endovascular solution to exsanguination from pelvic or torso haemorrhage. It should be considered where the patient presents in a peri-arrest state, or if there is likely to be a delay in providing rapid proximal haemorrhage control. In particular, in a Role 2 Afloat configured as a 2-1-2 facility, REBOA offers a means of temporising a second shocked patient, should the operating table be in current use and therefore unavailable. 
Box 1 - REBOA procedural guideline:

The following is a proposed as a guideline for performing REBOA in R2A

1. Confirm indication - exsanguinating torso/pelvic haemorrhage and critical or peri-arrest state. Theatre unavailable or loss of cardiac output during anaesthesia likely.

2. Open and prepare equipment, explain procedure to patient and team members.

3. Identify femoral artery pulsation, using ultrasound to confirm.

4. Infiltrate $10 \mathrm{ml} 1 \%$ lidocaine in skin and soft tissues around intended puncture site, $2 \mathrm{~cm}$ below inguinal ligament.

5. Make a generous stab-incision in the skin to allow passage of the sheath.

6. Insert $18 \mathrm{~g}$ introducer needle from MAC ${ }^{\mathrm{TM}}$ line set into femoral artery, fix needle in vessel with fingers, advance $0.035^{\prime \prime}$ guidewire, remove needle and insert Leadercath ${ }^{\text {TM }}$ catheter over-thewire. Confirm presence in artery using ultrasound. Remove guidewire to confirm arterial blood flow; NB - this may not appear very pulsatile in the setting of profound hypotension.

7. Re-insert $45 \mathrm{~cm}$ guidewire from $M C_{-}^{T M}$ line through Leadercath ${ }_{-}^{T M}$ device, remove Leadercath ${ }_{-}^{T M}$ ensuring the guidewire remains in the vessel. Insert MAC line over guidewire using forward twisting motion. If necessary, extend skin incision over guidewire. Remove MAC line dilator and guidewire. Flush and lock side-ports.

8. Insert 7F Syntel ${ }^{\mathrm{TM}}$ balloon through hydrostatic valve of MAC line. Insert to desired depth:

a. For Zone 1 REBOA - estimate length from CFA insertion site to midway between xiphoid process and suprasternal notch $(\sim 45 \mathrm{~cm})$.

b. For Zone 3 REBOA - estimate length from CFA insertion site to umbilicus ( $30 \mathrm{~cm})$.

9. Gently inflate Syntel ${ }_{-}^{\top M}$ balloon with $2.5 \mathrm{ml} 0.9 \%$ saline via 3-way tap, or until resistance is felt.

10. Lock the balloon using the 3-way tap.

11. Note time, and length of catheter at haemostatic valve of $\mathrm{MAC}_{-}^{\mathrm{TM}}$ line.

Post-REBOA actions:

Secure sheath with sutures.

Allocate team member to hold balloon shaft and avoid accidental catheter/sheath dislodgement or balloon deflation.

Commence blood product transfusion and prepare for surgery.

Insert arterial pressure monitoring line in right radial artery.

If arterial pressure supra-normal, release $0.5 \mathrm{ml}$ aliquots from-syntel balloon.

If surgery will take $>10$ mins, obtain plain anteroposterior X-ray to confirm placement. 


\section{References}

1. Hodgetts TJ, Kirkman E, Mahoney PF. UK defence medical services guidance for the use of recombinant factor VIIA (RFVIIA) in the deployed military setting. Journal of the Royal Army Medical Corps. 2007;153:299-300.

2. Midwinter MJ. Damage control surgery in the era of damage control resuscitation. Journal of the Royal Army Medical Corps. 2009;155:323-326.

3. Eastridge BJ, Mabry RL, Seguin P. Death on the battlefield (2001-2011): implications for the future of combat casualty care. The Journal of Trauma and Acute Care Surgery. 2012;73:431-437.

4. Penn-Barwell JG, Roberts, SAG, Midwinter, MJ. Improved survival in UK combat casualties from Iraq and Afghanistan: 2003-2012. Journal of Trauma and Acute Care Surgery. 2015;78:10141020.

5. Morrison JJ, America TER. Noncompressible torso hemorrhage: a review with contemporary definitions and management strategies. Surgical Clinics of North America. 2012;92:843-858.

6. Barnard EBG, Morrison JJ, Madureira RM. Resuscitative endovascular balloon occlusion of the aorta (REBOA): a population based gap analysis of trauma patients in England and Wales. Emergency Medicine Journal. 2015;32:926-932.

7. True NA, Siler S, Manning JE. Endovascular resuscitation techniques for severe hemorrhagic shock and traumatic arrest in the presurgical setting. Journal of Special Operations Medicine.

2013;13:33-37.

8. Avaro JP, Mardelle V, Roch A. Forty-minute endovascular aortic occlusion increases survival in an experimental model of uncontrolled hemorrhagic shock caused by abdominal trauma. Journal of Trauma and Acute Care Surgery. 2011;71:720-726.

9. Markov NP, Percival TJ, Morrison JJ, Ross JD, Scott DJ. Physiologic tolerance of descending thoracic aortic balloon occlusion in a swine model of hemorrhagic shock. Shock. 2013;153:848-856.

10. Morrison JJ, Ross JD, Houston RIV, Watson JDB. Use of resuscitative endovascular balloon occlusion of the aorta in a highly lethal model of noncompressible torso hemorrhage. Shock. 2014;41:130-137.

11. Morrison JJ, Percival TJ, Markov NP. Aortic balloon occlusion is effective in controlling pelvic hemorrhage. Journal of Surgical Research. 2012;177:341-347.

12. Gamberini E, Coccolini F. Resuscitative Endovascular Balloon Occlusion of the Aorta in trauma: a systematic review of the literature. World Journal of Emergency Surgery. 2017;12

13. DuBose JJ, Scalea TM. The AAST prospective Aortic Occlusion for Resuscitation in Trauma and Acute Care Surgery (AORTA) registry: data on contemporary utilization and outcomes of 
aortic occlusion and resuscitative endovascular balloon occlusion of the aorta (REBOA). Journal of Trauma and Acute Care Surgery. 2016;81:409-419.

14. Aso S, Matsui H, Fushimi, K. Resuscitative endovascular balloon occlusion of the aorta or resuscitative thoracotomy with aortic clamping for noncompressible torso hemorrhage: $\mathrm{A}$ retrospective nationwide study. Journal of Trauma and Acute Care Surgery. 2017;82:910-914.

15. Linnebur $\mathrm{M}$, Inaba $\mathrm{K}$, Haltmeier T. Emergent non-image-guided resuscitative endovascular balloon occlusion of the aorta (REBOA) catheter placement: A cadaver-based study. Journal of Trauma and Acute Care. 2016;81:453-457.

16. Okada Y, Narumiya H. Anatomical landmarks for safely implementing resuscitative balloon occlusion of the aorta (REBOA) in zone 1 without fluoroscopy. Scandinavian Journal of Trauma, Resuscitation and Emergency Medicine. 2017;25:1-5.

17. Russo RM, Williams TK, Grayson JK. Extending the golden hour: partial resuscitative endovascular balloon occlusion of the aorta in a highly lethal swine liver injury model. The journal of Trauma and Acute Care Surgery. 2016;80:372-380.

18. Russo RM, Neff LP, Lamb CM. Partial resuscitative endovascular balloon occlusion of the aorta in swine model of hemorrhagic shock. Journal of the American College of Surgeons.

2016;223:359-368.

19. Matsumura Y, Matsumoto J, Kondo H, Idoguchi JK. Fewer REBOA complications with smaller devices and partial occlusion: evidence from a multicentre registry in Japan. Emergency Medicine Journal. 20161-7.

20. Larzon T, Geijer H, Gruber, G. Fascia suturing of large access sites after endovascular treatment of aortic aneurysms and dissections. Journal of Endovascular Therapy. 2006;13:152-157.

21. Sadek S, Lockey DJ, Lendrum RA, Perkins Z, Price J. Resuscitative endovascular balloon occlusion of the aorta (REBOA) in the pre-hospital setting: an additional resuscitation option for uncontrolled catastrophic haemorrhage. Resuscitation. 2016;107:135-138.

22. Smith SA, Hilsden R, Beckett A. The future of resuscitative endovascular balloon occlusion in combat operations. Journal of The Royal Army Medical Corps. 2017;163:296-300.

23. Manning JE, Murphy CA, Hertz CM. Selective aortic arch perfusion during cardiac arrest: a new resuscitation technique. Annals of Emeregency Medicine. 1992;21:1058-1065.

24. Tisherman SA, Brenner ML. Taking advanced endovascular techniques out of the hospital: Ready for prime time. Resuscitation. 2016;107:A3-A4.

25. Kutcher ME, Forsythe RM, Tisherman SA. Emergency preservation and resuscitation for cardiac arrest from trauma. International Journal of Surgery. 2016;33:209-212.

\section{Contributorship Statement}

All authors took an equal role in discussion of the concepts, composition, generation and review of the manuscript.

\section{Competing interests}

No, there are no competing interests 